\title{
Estudio comparativo del rendimiento académico y la actividad física en dos institutos de enseñanza secundaria de Andalucía (España)
}

\section{Comparative study of academic achievement and physical activity in two secondary education institutes of Andalucia (Spain)}

\author{
Villena Serrano, M.. ${ }^{*}$, Castro López, R. ${ }^{2}$, Moreno Pulido, R. ${ }^{3}$ y Cachon Zagalaz, J. \\ 1 Licenciado CCAFD \\ 2 Universidad Internacional de La Rioja, Logroño, España \\ 3 Maestra Educación Física \\ 4 Universidad de Jaén, Jaén, España
}

\begin{abstract}
Resumen: Objetivo: Valorar si existe alguna relación entre el rendimiento académico y la condición física en un estudio realizado en dos centros educativos con características similares. Material y Método: Estudio experimental cuantitativo, en el que han participado 144 alumnos de ambos sexos de edades comprendidas entre los 14 y los 18 ańos que cursan $4^{\circ}$ curso de Enseñanza Secundaria Obligatoria (ESO). Se ha analizado la condición física mediante test estandarizados donde se han recogido datos referentes a la resistencia (test de Cooper), velocidad (test de 50 metros lisos), flexibilidad (test de flexión profunda de tronco) y fuerza abdominal (test de abdominales por minuto). Resultados y Conclusiones: Los resultados muestran una relación estadísticamente significativa entre el rendimiento académico y la actividad física de los alumnos en las pruebas de resistencia, velocidad y flexibilidad, aunque los mismos no han determinado relación significativa entre el rendimiento académico y la prueba de abdominales por minuto.
\end{abstract}

Palabras clave: Educación, Condición física, Test estandarizados, Eurofit. Abstract: Objective: To assess whether there is some relationship between academic performance and physical condition, a study based on two schools with similar features. Methodology and Method: Quantitative experimental study, in which participated 144 pupils of both sexes and aged between 14 and 18 years who were in the 4th year of Obligatory Secondary Education (ESO). It was analyzed using standardized physical fitness test and have collected data on resistance (Cooper test), speed (50 meters sprint test), flexibility (test of deep trunk flexion) and abdominal strength (abs test for minute). Results and conclusion: There is a significant relationship between academic performance and physical activity of students in the data of resistance, speed and flexibility, although there is no significant relationship between academic performance and test Abs per minute. Keywords: Education, physical condition, Standardized Test, Eurofit.

\section{Introducción}

A lo largo de la historia, la educación física (EF) en España ha sufrido numerosos cambios, posicionándose como indica Martínez (2000, p. 83), “en una posición relativa más favorable que otras épocas, disponiendo de un conocimiento que tal vez nos permita superar la circularidad que ha tenido la historia de la EF”. Sin embargo, y contrariamente, diferentes autores ańaden que actualmente se está viviendo un intento de reemplazar las clases de EF para aumentar el logro académico de los estudiantes. A pesar de esta tendencia, hay una clara evidencia que indica que el rendimiento académico mejorará si no se cortan las clases de EF (Coe, Malina, Pivarnik, Reeves y Womack, 2005).

De los artículos analizados se han detectado numerosos estudios con una relación positiva entre logros académicos y la actividad física (AF) (Lavallee y Shephard, 1994; Etnier y Sibley, 2003; Tomporowski, 2003; Blizzard, Dean, Dwyer, Lazarus y Sallis, 2001). Otros sin embargo, han demostrado que no existe ninguna correlación (Daley y Ryan, 2000) o una relación inversa (Inman, Tremblay y Willms, 2000).

Dirección para correspondencia [Correspodence address]: Manuel Villena Serrano. E-mail: m_villena_serrano@yahoo.es
Siendo más específicos y analizando la relación entre condición física y rendimiento académico, se han encontrado diversos estudios en los que se determinan algunas variables de la condición física que influye de una manera u otra al rendimiento académico.

En relación con los parámetros psicológicos del alumnado, la a AF además de provocar un aumento rendimiento académico, provoca una mejora la motivación y la reducción del aburrimiento. Lo que puede conducir a aumento de la capacidad de atención y concentración (Coe et al., 2005). Además, el aumento de los niveles de AF también puede estar relacionados con el aumento de la autoestima, que se podría esperar un mejor comportamiento en clase por parte del alumnado (Shephard, 1996, p. 32).

Como trabajos más relevantes en la materia se encuentra el estudio de Caterino y Polak (1999) donde encuestaron a 383 directores escolares sobre el tiempo de recreo. Los análisis indicaron que el período de recreo es subjetivamente saludable y productivo para los niños. De hecho, "la mayor parte de los encuestados creen que los nińos que tienen mayor tiempo de recreo están más alerta y más relajados durante el día” (Caterino y Polak, 1999, p. 216). Además, Pellegrini 
(1991) explica cómo proporcionar un cambio en el ambiente de la clase, contrarrestando el estado de aburrimiento de los alumnos. Estos cambios implican que atención a las tareas del aula puede ser recuperada a través de la activación en el tiempo de recreo. Según Caterino y Polak (1999) los directores, consejeros y educadores parecen creer que la AF puede mejorar la atención y ayuda a los niños a concentrarse en las tareas académicas.

Continuando con la revisión bibliográfica se encuentran Aznar y Webster (2006, p. 76) los cuales afirman que "existen pruebas científicas que demuestran que un incremento significativo del tiempo escolar dedicado a la EF relacionada con la salud no tiene efectos perjudiciales para el rendimiento académico del alumnado sino que proporciona importantes beneficios para la salud". Además, estos mismos autores ańaden que se ha demostrado que los niños y niñas con niveles de AF más elevados tienen mayores posibilidades de desarrollar un mejor funcionamiento cognitivo (Aznar y Webster, 2006).

Teniendo en cuenta los trabajos revisados, puede afirmar en términos generales, que la literatura científica indica que el aumento de la EF, generan un efecto positivo, más que negativo, sobre el rendimiento académico de niños y niñas adolescentes, en palabras de Wi-Young (2013, p. 1) "los estudios que han investigado la relación entre la AF y la salud, mostraron que la AF regular puede mejorar el rendimiento académico en los estudiantes adolescentes". La AF tiene numerosos beneficios tanto físicos, control de peso, hueso y fuerza muscular; como psicológicos, salud mental y el estado de ánimo en relación con las actividades diarias (Wi-Young, 2013).

Siguiendo la línea de los anteriores, Inman, Tremblay y Willms (2000, p. 2) indican que "la participación regular en AF mejora nuestra salud además de reducir el riesgo de diabetes tipo II, hipertensión y colesterol”. Por tanto, la relación entre la AF y la salud es bastante evidente en el ámbito científico, sin embargo, el nexo de unión entre el rendimiento académico y la AF no está tan claro.

Con este estudio se va a defender la hipótesis de la existencia de una relación entre el rendimiento académico (tomando como nota media las asignaturas comunes) y la AF (medida mediantes test estandarizados de condición física), haciendo diferenciaciones entre centros, sexos e intra-grupos tanto de sexo masculino como de sexo femenino de cada centro analizado.

\section{Material y Método}

\section{Variables}

Se ha realizado el análisis de dos variables: rendimiento académico (dependiente), la cual ha sido medida mediante la nota media de las asignaturas comunes de los alumnos de $4^{\circ}$ curso de Educación Secundaria Obligatoria (ESO) de ambos centros. En segundo lugar, la variable AF (independiente) que se ha medido mediante test de condición física estandarizados. Los test seleccionados son los que más se han adecuado a las características y los materiales de los centros.

\section{Muestra}

Los participantes de este estudio fueron un grupo de 144 alumnos (varones y mujeres) de dos centros educativos de ESO, pertenecientes al $4^{\circ}$ curso y con edades comprendidas entre 14 y 18 ańos. Todos escolarizados en el nivel educativo de $4^{\circ}$ de ESO en dos centros educativos de zonas urbanas de Andalucía (España).

El grupo de alumnos del centro educativo 1 se compone de 84 alumnos (55.55\%) de los cuales 36 son varones y 38 mujeres. Por otro lado el grupo de alumnos del centro educativo 2 se compone de 60 alumnos (44.45\%) de los cuales 28 son varones y 32 mujeres.

\section{Instrumento/Materiales}

En el trabajo de campo, es decir, en la medición de la condición física se han utilizado diferentes test estandarizados (Batería Eurofit adaptada, Test de Cooper, test de $50 \mathrm{~m}$ lisos). Por otro lado, en el análisis del rendimiento académico hemos utilizado la nota media de las asignaturas comunes de los alumnos. Finalmente en el análisis estadístico de los datos obtenidos en cada una de las pruebas se empleó el programa estadístico SPSS 20 (Windows).

Los materiales usados en los test de condición física han sido los mismos en ambos centros educativos, aunque las instalaciones han sido diferentes.

En el análisis del rendimiento académico de los alumnos, se va a utilizar la nota media de las asignaturas comunes de los alumnos de $4^{\circ}$ curso de ESO correspondientes a la segunda evaluación, ya que el comienzo de este estudio fue en la $3^{\circ}$ evaluación. Las asignaturas a analizar han sido: Lengua y Literatura; Ciencias Sociales, Geografía e Historia; Inglés, EF y Educación Ético Cívica.

En el análisis de la variable independiente (condición física), hemos seleccionado los test que más se adaptan a las características de los alumnos y a los materiales disponibles para la realización de las pruebas en ambos centros.

Los test seleccionados han sido: el test de Cooper, para la medición de la condición física (test de resistencia cardiorrespiratoria de carrera); la prueba de flexión profunda de tronco mide la amplitud musculo-articular; el test de velocidad de $50 \mathrm{~m}$ lisos es un test que mide la velocidad de desplazamiento y la fuerza explosiva de los sujetos; y por último, el Test de fuerza abdominal. Es un test que mide la potencia del tronco 


\section{Procedimiento}

Antes de comenzar el trabajo de campo, se ha informado a ambos centros de que se iba a realizar un estudio con los alumnos de $4^{\circ}$ curso de ESO y seguidamente, se habló con los alumnos y las familias para informar de que se va a realizar un estudio comparativo entre las variables rendimiento académico y la AF para que presten su consentimiento en la realización de las actividades a desarrollar dentro del estudio, en el cual, todos los alumnos han deseado participar. En ambos centros se han intentado disminuir las diferencias para que el margen de error sea el mínimo.

El orden de las pruebas ha sido el siguiente:

- Día 1: Prueba de abdominales y prueba de velocidad.

- Día 2: Prueba de flexibilidad y test de Cooper. Antes de cada prueba se explica a todos los alumnos el desarrollo del test, su realización correcta y las instrucciones necesarias para el desarrollo de la misma.

Todos los datos han sido recogidos en una hoja de control donde aparecen los ítems de: Centro escolar, curso/clase, número de alumno, nota media en las asignaturas, resultado abdominales por minuto, resultado $50 \mathrm{~m}$, resultado flexión profunda de tronco, resultado test de Cooper.

\section{Resultados}

Los resultados se exponen de modo en que se observen los parámetros analizados de diferentes formas.

En primer lugar, como propósito principal de este trabajo, se van a analizar los resultados para establecer la relación entre los parámetros de rendimiento académico y de condición física. Una vez analizados estos parámetros, se pasará a realizar un análisis comparativo entre los centros, entre géneros, intra-grupo masculino e intra-grupo femenino como segundo propósito de este trabajo.

En cuanto al análisis sobre la relación entre rendimiento académico y condición física, se van a realizar diferentes pruebas estadísticas de análisis de datos pasa saber si existe relación entre ambas variables. La prueba más significativa para establecer relación entre variables es la prueba ANOVA.

En el análisis estadístico de los datos, se quiere comprobar si existe alguna relación entra las variables de AF y la variable nota media. Para saber si existen, o no, diferencias significativas entre ambos grupos hay que realizar un ANOVA.

Al realizar un ANOVA, debemos saber si la variable dependiente sigue una distribución normal, lo que nos demostrará si en el test ANOVA existe fiabilidad o no. En la prueba de normalidad el P valor Sig. es mayor a .05, es decir la nota media sigue una distribución normal. Como la muestra es mayor a $\mathrm{n}>50$ los resultados a analizar son los de Kolmogorov-Smirnov.

Tabla 1. Nota media.

\begin{tabular}{lccccccccc}
\hline & \multicolumn{3}{c}{ Centro 1 } & \multicolumn{3}{c}{ Centro 2 } & \multicolumn{3}{c}{ Total } \\
\cline { 2 - 12 } & Hombres & Mujeres & Total & Hombres & Mujeres & Total & Hombres & Mujeres & Total \\
\hline Nota Media & 6.32 & 6.52 & 6.42 & 5.58 & 5.93 & 5.75 & 5.95 & 6.22 & 6.08 \\
\hline
\end{tabular}

Tabla 2. Prueba de normalidad variable Nota Media.

\begin{tabular}{cccccccc}
\hline & \multicolumn{3}{c}{ Kolmogorov-Smirnova } & \multicolumn{3}{c}{ Shapiro-Wilk } \\
\cline { 2 - 7 } & Estadístico & gl & Sig. & Estadístico & gl & Sig. \\
\hline Nota Media & .037 & 144 & $.200^{*}$ & .994 & 144 & .857 \\
\hline
\end{tabular}

Cuando una muestra procede de una población normal, los puntos correspondientes a cada par se encuentran agrupados en torno a la diagonal representada en el diagrama. Las desviaciones de la diagonal indican desviaciones de la normalidad. Como vemos en este caso podríamos decir que sí se distribuye de manera Normal.

Figura 1. Grafico Q-Q normal de Nota media.

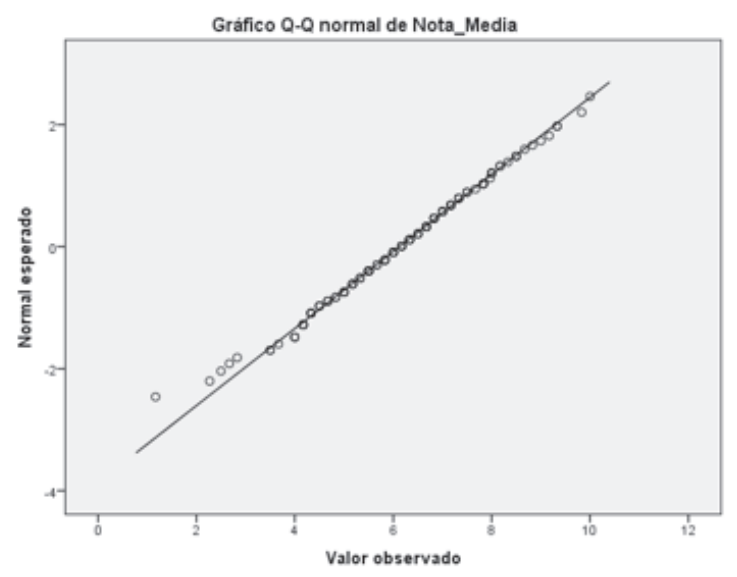

Estudio comparativo del rendimiento académico y la actividad física en dos... SporTK, 4(2), 11-18 
Al realizar la prueba ANOVA queremos encontrar si existen relaciones significativas en las variables. Si existen relaciones significativas (valores >.05) aceptaremos la hipótesis de que existe relación entre la AF y el rendimiento académico. Si al realizar la prueba ANOVA se obtiene una significación baja (valores < .05) rechazamos la hipótesis de que exista relación alguna.

En todas las pruebas se obtienen datos superiores a .05 menos en la prueba de test de abdominales donde el resultado es menor a .05 por lo que no existe relación significativa entre este test y el rendimiento académico en los resultados analizados.

En el resto de prueba se obtienen resultados bastante elevados lo que proporciona un alto nivel de relación entre ambas variables.

Tabla 3. ANOVA Variable dependiente nota media y pruebas de rendimiento físico.

\begin{tabular}{|c|c|c|c|c|c|c|}
\hline & & Suma de cuadrados & gl & Media cuadrática & $\mathrm{F}$ & Sig. \\
\hline \multirow{3}{*}{ Flexibilidad } & Inter-grupos & 1970.134 & 42 & 46.908 & .847 & .724 \\
\hline & Intra-grupos & 5595.192 & 101 & 55.398 & & \\
\hline & Total & 7565.326 & 143 & & & \\
\hline \multirow{3}{*}{ Abdominales } & Inter-grupos & 5442.804 & 42 & 129.591 & 1.884 & .005 \\
\hline & Intra-grupos & 6947.856 & 101 & 68.791 & & \\
\hline & Total & 1239.660 & 143 & & & \\
\hline \multirow{3}{*}{ Velocidad } & Inter-grupos & 50.222 & 42 & 1.196 & .655 & .938 \\
\hline & Intra-grupos & 184.505 & 101 & 1.827 & & \\
\hline & Total & 234.726 & 143 & & & \\
\hline \multirow{3}{*}{ Resistencia } & Inter-grupos & 6405748.016 & 42 & 152517.810 & 1.030 & .440 \\
\hline & Intra-grupos & 14950640.873 & 101 & 148026.147 & & \\
\hline & Total & 21356388.889 & 143 & & & \\
\hline
\end{tabular}

En el análisis comparativo entre centros, se van a utilizar en primer lugar, los datos de los test ANOVA para ver la relación existente en diferentes centros en las variables rendimiento académico y AF (propósito de este trabajo). Una vez analizado estos datos, se procederá a comparar las variables una a una teniendo en cuenta la variable nota media (media de asignaturas comunes) y variable AF (prueba de flexibilidad, de abdominales, de resistencia y de velocidad).

En la tabla 3 se pueden observar los resultados del test ANOVA donde se ha comparado la nota media con cada una de las pruebas físicas realizadas en el centro divididas entre centros. En los resultados se pueden observar que todos los resultados existen relaciones significativas (valores $>.05$ ) por lo que hay relación entre la variable nota media y las pruebas físicas realizadas para analizar la AF de los alumnos.

El Centro 1 presenta resultados superiores en la correlación de la prueba de abdominales y en la de flexibilidad; mientras que el Centro 2 presenta resultados superiores en la correlación de la prueba de flexibilidad y en la de resistencia.
Tabla 4. Comparación ANOVA de un factor pruebas de rendimiento físico con la nota media como variable dependiente Centro 1 y Centro 2 .

\begin{tabular}{llcc}
\hline & & $\begin{array}{c}\text { Centro 1 } \\
\text { Sig. }\end{array}$ & $\begin{array}{c}\text { Centro 2 } \\
\text { Sig. }\end{array}$ \\
\hline Flexibilidad & Inter-grupos & .739 & .905 \\
Abdominales & Inter-grupos & .155 & .056 \\
Resistencia & Inter-grupos & .378 & .774 \\
Velocidad & Inter-grupos & .981 & .770 \\
\hline
\end{tabular}

En cuanto al análisis comparativo de género, se van a utilizar en primer lugar los datos de los test ANOVA para ver la relación existente en los sexos sin hacer diferenciación entre centros en las variables rendimiento académico y AF (propósito de este trabajo). Una vez analizado estos datos, se procederá a comparar las variables una a una teniendo en cuenta la variable nota media (media de asignaturas comunes) y variable AF (prueba de flexibilidad, de abdominales, de resistencia y de velocidad).

En la tabla 4 se pueden observar los resultados del test ANOVA donde se ha comparado la nota media con cada una de las pruebas físicas realizadas en el centro divididas por sexos. En los resultados se pueden observar que no todos los resultados existen relaciones significativas (valores $>.05$ ) por 
lo que no hay relación entre todas las variables de las pruebas físicas y la variable nota media.

Como se ha podido observar en la tabla 1 el resultado del test de abdominales de la comparación de datos ANOVA general tampoco presenta relaciones significativas al igual que ocurre en la relación de Abdominales con nota media en el grupo de mujeres.

El resto de resultados sí presentan relación significativa. En el test de flexibilidad y en el test de velocidad se obtienen datos con mayor relación en las mujeres que en los hombres. Mientras que en la prueba de resistencia y en la prueba de abdominales se obtienen resultados superiores para el grupo de hombres.

Tabla 5. Comparación ANOVA de un factor pruebas de rendimiento físico con la nota media como variable dependiente entre sexos de ambos centros

\begin{tabular}{llcc}
\hline & & $\begin{array}{c}\text { Hombres } \\
\text { Sig. }\end{array}$ & $\begin{array}{c}\text { Mujeres } \\
\text { Sig. }\end{array}$ \\
\hline Flexibilidad & Inter-grupos & .636 & .814 \\
Abdominales & Inter-grupos & .333 & .011 \\
Resistencia & Inter-grupos & .987 & .503 \\
Velocidad & Inter-grupos & .787 & .934 \\
\hline
\end{tabular}

En el análisis comparativo entre el grupo masculino de ambos centros, se van a utilizar en primer lugar los datos de los test ANOVA para ver la relación existente en el sexo masculino haciendo una diferenciación entre centros en las variables rendimiento académico y AF (propósito de este trabajo). Una vez analizado estos datos, se procederá a comparar las variables una a una teniendo en cuenta la variable nota media (media de asignaturas comunes) y variable AF (prueba de flexibilidad, de abdominales, de resistencia y de velocidad).

En la tabla 5 se pueden observar los resultados del test ANOVA donde se ha comparado la nota media con cada una de las pruebas físicas realizadas en el centro divididas por centros y entre sexos. Como en las tabla 3 y 4 no en todos los resultados existe relaciones significativas (valores >.05) por lo que no hay relación entre la todas las variables de la condición física analizadas y la nota media de los alumnos.

Como se pudo observar anteriormente, en el test de abdominales, no existía relación significativa. Aquí en esta nueva comparación vuelve a ocurrir lo mismo para el grupo de hombres del Centro 2.

Como se puede apreciar el Centro 1 presenta resultados superiores en la correlación de todas las pruebas analizadas. Es decir, existe una mayor relación en la hipótesis de que existe relación entre el rendimiento académico y la AF de los alumnos analizados.
Tabla 6. Comparación ANOVA de un factor. Pruebas de rendimiento físico con la nota media como variable dependiente entre el sexo masculino de cada centro.

\begin{tabular}{llcc}
\hline & & \multicolumn{2}{c}{ Hombres } \\
\cline { 3 - 4 } & & $\begin{array}{c}\text { Centro 1 } \\
\text { Sig. }\end{array}$ & $\begin{array}{c}\text { Centro 2 } \\
\text { Sig. }\end{array}$ \\
\hline Flexibilidad & Inter-grupos & .727 & .703 \\
Abdominales & Inter-grupos & .744 & .015 \\
Resistencia & Inter-grupos & .969 & .696 \\
Velocidad & Inter-grupos & .977 & .566 \\
\hline
\end{tabular}

Para el análisis comparativo entre el grupo femenino de ambos centros, se van a utilizar en primer lugar los datos de los test ANOVA para ver la relación existente en el sexo femenino haciendo una diferenciación entre centros en las variables rendimiento académico y AF (propósito de este trabajo). Una vez analizado estos datos, se procederá a comparar las variables una a una teniendo en cuenta la variable nota media (media de asignaturas comunes) y variable AF (prueba de flexibilidad, de abdominales, de resistencia y de velocidad).

En la tabla 6 se pueden observar los resultados del test ANOVA donde se ha comparado la nota media con cada una de las pruebas físicas realizadas en el centro divididas por centros y entre sexos. Como excepción a las tablas anteriores de las diferentes comparativas de los test ANOVA en la tabla 3,4 y 5 no en todos los resultados existían relaciones significativas (valores $>.05$ ). En cambio, en esta tabla, la tabla número 6 encontramos relaciones significativas para todos los valores de la condición física.

Como se puede apreciar el Centro 1 presenta resultados superiores en la correlación de las pruebas de flexibilidad, y velocidad, mientras que el Centro 2, presenta correlación superior en el test de abdominales y en la prueba de resistencia.

Tabla 7. Comparación ANOVA de un factor. Pruebas de rendimiento físico con la nota media como variable dependiente entre el sexo femenino de cada centro

\begin{tabular}{llcc}
\hline & \multicolumn{2}{c}{ Mujeres } \\
\cline { 3 - 4 } & & Centro 1 & Centro 2 \\
& & Sig. & Sig. \\
\hline Flexibilidad & Inter-grupos & .674 & .624 \\
Abdominales & Inter-grupos & .092 & .125 \\
Resistencia & Inter-grupos & .134 & .921 \\
Velocidad & Inter-grupos & .963 & .774 \\
\hline
\end{tabular}

\section{Discusión}

El objetivo de este estudio fue investigar la relación entre AF y rendimiento académico en adolescentes de $4^{\circ}$ curso de la ESO en dos diferentes regiones de la comarca de Andalucía 
(España). Los principales resultados mostraron que sí existe una correlación entre el rendimiento académico y la AF en todos sus análisis, excepto con la relación de la prueba de abdominales. Tanto en el análisis del grupo general (análisis de la correlación de todos los datos) como en el resto de análisis desarrollados en este trabajo, obtuvo una relación no significativa o muy poco significativa entre el rendimiento académico y la prueba de abdominales (.005). En el resto de pruebas, obtenemos resultados con alta significatividad, llamando la atención el dato de una correlación de .938 entre el rendimiento académico y la prueba de velocidad.

Este estudio corrobora lo analizado en diferentes trabajos revisados sobre tal temática. En el trabajo de Wi-Young (2013) donde se recogieron datos de 75.066 estudiantes adolescentes con un cuestionario donde se midieron entre otros el tipo de actividad física que realizaba dividida en vigorosa o moderada, y la frecuencia con la que se realizaba. Los resultados mostraron que la AF vigorosa se correlacionó positivamente con el rendimiento académico en el caso de los niños, y la AF moderada se relacionó positivamente con el rendimiento académico de los niños y las niñas. Como dato relevante de este trabajo se concluyó que el ejercicio aumenta la saturación de oxígeno y la angiogénesis en las regiones del cerebro esenciales para la realización de tareas (Wi-Young, 2013). En segundo lugar, este mismo autor, también indica que el ejercicio físico aumenta la actividad de los neurotransmisores como la serotonina y la norepinefrina en el cerebro facilitando un procesamiento de la información (Wi-Young, 2013).

Al igual, el estudio de Brown et al. (2008). Se observó un pequeño beneficio, significativo, para el rendimiento académico, en matemáticas y lectura, para las niñas que realizan AF. Cantidades de AF no afectan negativamente al logro académico de los alumnos. Las preocupaciones acerca de los efectos adversos sobre el rendimiento no son razones para legitimar los programas de EF.

Como en el estudio de Brown et al. (2008) y el de WiYoung (2013), se observa una existente relación positiva entre la variable rendimiento académico y la variable AF en diferentes artículos revisados: Lavallee y Shephard (1994) Etnier y Sibley (2003) Tomporowski (2003) Blizzard, Dean, Dwyer, Lazarus y Sallis (2001)

Con valor neutro en esta temática, es decir, que indica que no existe relación entre la variable rendimiento académico y AF, se encuentra el trabajo de Daley y Ryan (2000). Este estudio analizó la relación entre el desempeño académico (notas de Inglés, Matemáticas y Ciencias) y la participación en la AF de los adolescentes. En los resultados, no se encontraron correlaciones significativas, aunque se registraron correlaciones negativas débiles entre la cantidad de tiempo (en minutos) en el deporte y el ejercicio, y las puntuaciones de inglés y ciencias para niños.
En contraposición a la relación positiva, el estudio de Inman, Tremblay y Willms (2000) indica que hay una relación inversa entre ambas variables. Este estudio examinó la relación entre la AF y con las puntuaciones en matemáticas. La relación entre la masa corporal tenía una relación negativa con la AF, y por otro lado, la AF afectaba positivamente a la autoestima.

En resumen, este estudio ha demostrado mediante los datos analizados que existe una relación positiva entre el rendimiento académico y la AF. El análisis revela que ambos sexos, los cuales tienen una AF moderada, consiguen niveles más elevados de notas en las asignaturas analizadas en este trabajo.

\section{Conclusiones}

Los resultados han mostrados que la AF se correlaciona en general positivamente con el rendimiento académico (Tabla 3), utilizando como ítems la resistencia medida mediante el test de Cooper, la velocidad medida mediante la prueba de 50 metros lisos, la flexibilidad medida mediante el test de flexión profunda de tronco y finalmente la fuerza abdominal medida con el test de abdominales por minuto. En la prueba de abdominales no existe relación significativa con la nota media mediante los datos analizados.

En el resto de análisis, encontramos que en la comparación entre centros (Tabla 4) se puede apreciar diferencias de correlación de las pruebas entre el Centro 1 y el Centro 2. Estos valores pueden ser causados por el tipo de unidad didáctica que se estuviera desarrollando en el momento de la recogida de datos.

Seguidamente en el análisis de comparación entre sexos (Tabla 5). Principalmente, los resultados muestran una mayor puntuación de correlación de la significatividad de los resultados en alumnos de sexo masculino en las pruebas físicas que en el sexo femenino. Sin embargo, existe una mayor puntuación de la nota media con valores mayores para el grupo de sexo femenino (6.22) sobre el sexo masculino (5.95). Estos valores indican que existe una correlación entre el rendimiento académico y la actividad física, pero no, que aquel alumno que realice más actividad física sea el que mejores resultados académicos va a obtener.

En la diferenciación inter- grupo del sexo masculino (Tabla 6) y sexo femenino (Tabla 7) entre los dos centros en las pruebas de flexibilidad, abdominales y resistencia. El grupo masculino del Centro 2 tiene una cierta superioridad sobre el Centro 1. Por otro lado, el Centro 1 presenta una cierta superioridad en la nota media y en la prueba de velocidad frente al instituto Centro 2. El inter-grupo femenino los resultados bastante similares al grupo de hombres. Destacamos la similitud en los resultados, especialmente en la prueba de resistencia, donde el inter-grupo femenino presenta resultados muy similares. Estos resultados pueden ser debidos a que 
los centros desarrollen unidades didácticas diferentes dentro de la programación de centro, o simplemente, el tipo de ac- tividad física que desarrolle cada uno de los alumnos como actividades extraescolares.

\section{Referencias bibliográficas}

1. Aznar, S., Webster, T. (2006). Actividad física y salud en la infancia y en la adolescencia: Guía para las personas que participan en su educación. Madrid: Ministerio de Educación y Ciencia.

2. Blizzard, J., Dean, K., Dwyer, T., Lazarus, R., Sallis, J. (2001). Relationship of academic performance to physical activity and fitness in children. Pediatric Exercise Science, 13, 225-237.

3. Brown, D., Carlson, S., Diezt, W., Fulton, J., Kohl, H., Lee, S., Maynard, M. (2008). Physical Education and Academic Achievement in Elementary School: Data From the Early Childhood Longitudinal Study. Am J Public Health, 98 (4), 721-727.

4. Cameron, C., Craig, C., Tudor, C. (2013). Relationship between parent and child pedometer-determined physical activity: a sub-study of the CANPLAY surveillance study. International Journal of Behavioral Nutrition and Physical Activity, 18, 8-10.

5. Caterino, M., Polak, E. (1994). The effects of three types of activity on the performance of a test of concentration by fourth grade children. 10 zua Jorrrnal of Health, Physical Edrrcation, Recreation atid Dance, 27(2), 28-29.

6. Caterino, M., Polak, E. (1999). Effects of two types of activity on the performance of second-, third-and fourth-grade students on a test of concentration. Perceptual and Motor Skills, 89, 215-248.

7. Coe, D.P., Malina, R.M., Pivarnik, JM., Reeves, M.J., Womack, C. J. (2005). Effect of Physical Education and Activity Levels on Academic Achievement in Children. Medicine \& science in sports \& exercise. Recuperado el 09/06/2014 de http://www.wafapower.com/scienceresearch/ pe_academic_achievement.pdf

8. Daley, A., Ryan, D. (2000). Academic performance and participation in physical activity by secondary school adolescents. Perceptual and Motor Skills, 91, 531-553.

9. Etnier, J., Sibley, B. (2003). The relationship between physical activity and cognition in children: a meta-analysis. Pediatric Exercise Science, 15, 243-253.

10. Inman, J., Tremblay, M., Willms, D. (2000). The relationship between physical activity, self-esteem, and academic achievement in 12-year-old children. Pediatric exercise science, 12, 312-323. http://extranet.nuorisuomi.fi/download/attachments/3245041/the+relationship+between+ physical+activity,self-esteem,+and+academic+achievement+in+12-yearold+children.pdf. Recuperado el 09/06/2014.

11. Lavallee, H., Shephard, R. (1994). Academic skills and required physical education: the Trois Rivieres experience. Res. Suppl. 1, 1-12.

12. Martínez, L. (2000). A vueltas con la historia: una Mirada a la educación física escolar del S. XX. Revista de Educación, (extraordinario), 83-112.

13. Pellecrini, A. (1991). Outdoor recess: is it really necessary? Prirzcipal, $70(5), 40$.

14. Shephard, R. (1996). Habitual physical activity and academic performance. Nutr. Rev., 54, 32-35.

15. Tomporowski, P. (2003). Cognitive and behavioral responses to acute exercise in youths: a review. Pediatric Exercise Science, 15, 348-359.

16. Wi-Young, S. (2013). Association between physical activity and academic performance in Korean adolescent students. BMC Public Health 12(25-28). Recuperado el 09/06/2014 de http://www.biomedcentral. com/content/pdf/1471-2458-12-258.pdf. 